\title{
ERROR PERFORMANCE ANALYSIS USING COOPERATIVE CONTENTION-BASED ROUTING IN WIRELESS ADHOC NETWORKS
}

\author{
G.SRIMATHY \\ M.E/Communication Systems, S.A Engineering College, \\ Chennai-600 077, Tamil Nadu, India. \\ mail id: sri.mathy73@gmail.com
}

\begin{abstract}
In Wireless Ad hoc network, cooperation of nodes can be achieved by more interactions at higher protocol layers, particularly the MAC (Medium Access Control) and network layers play vital role. MAC facilitates a routing protocol based on position location of nodes at network layer specially known as Beacon-less geographic routing (BLGR) using Contention-based selection process. This paper proposes two levels of cross-layer framework - a MAC network cross-layer design for forwarder selection (or routing) and a MAC-PHY for relay selection. Wireless networks suffers huge number of communication at the same time leads to increase in collision and energy consumption; hence focused on new Contention access method that uses a dynamical change of channel access probability which can reduce the number of contention times and collisions. Simulation result demonstrates the best Relay selection and the comparative of direct mode with the cooperative networks. And also demonstrates the Performance evaluation of contention probability with Collision avoidance.
\end{abstract}

\section{KEY WORDS}

Ad hoc networks, relay selection, cross-layer design, geographic routing, cooperative networks.

\section{INTRODUCTION}

Ad Hoc wireless network which means the users or nodes that want to communicate with each other form self organising temporary network without any centralized administrator. Ad Hoc - a Latin phrase which means "for this purpose" (i.e.) no advance planning [1] [2]. An autonomous system of mobile hosts connected by wireless links, called Mobile Ad hoc NETworks (MANETs) [1] [5]. To achieve efficient communication benefits from cooperation, more interactions at higher protocol layers, particularly the MAC (Medium Access Control) [2] [9] and network layers, are vitally required. For this purpose, Beacon-less geographic routing (BLGR) [1] [3], based on position location of nodes and contention process.

\subsection{MAC in multihop Ad hoc networks:}

Ad Hoc networks consist of small, inexpensive, resource constrained nodes/host/router that communicate wirelessly in a multihop network [2]. Each node collaborates with other devices in the network to perform some operation for the end user, such as environmental monitoring or target tracking. End users typically desire to deploy nodes randomly throughout the target area in

DOI : 10.5121/ijcseit.2012.2211 
large numbers hundreds to thousands of nodes; however, some special cases may require the precise deployment of a smaller network.

Nodes communicate via multihop network [2] [3] to forward messages to the destination. Despite using it is to reduce energy requirements for communication, the wireless transceiver often consumes the largest amount of energy-per time period of use-within a node and, thus, provides the greatest potential for energy savings. Beyond improving the radio design, an efficient Medium Access Control (MAC) protocol possesses the greatest capability to decrease the energy consumption of the transceiver since it directly controls the transceiver operation.

In Section II, the paper summarizes related work. Section III, presents the proposed CoopGeo [1] with the cross-layer design for cooperative networks with the problem statement. Section IV gives the network model using BLGR, in which beaconless geographic routing and relay selection, along with it includes the protocol description. Section V, discusses the simulation results and evaluate its performance [1]. Finally, the paper concludes in section VI with future outlook.

\subsection{BLGR Concept}

BLGR is one of the most efficient and scalable routing solutions for wireless ad hoc and sensor networks. The key advantage of BLGR is that it needs neither the prior knowledge of network topology for making a route decision nor the periodic exchange of control messages (i.e., beacons) for acquiring neighbours' geographic locations. A current node can make its own routing decisions by using local information. In general, a BLGR protocol comprises two operating phases: forwarding phase and recovery phase. A forwarding node executes the greedy mechanism in the forwarding phase, and, if failing, switches to recovery mode to perform a face routing algorithm, finding another path to the destination.

\section{RELATED WORK}

Ad-hoc networks are infrastructure-less networks, auto configured with limited power Bandwidth. Each node participating in the network acts as both host and a router to forward packets for other nodes. Two nodes that are within transmission range of each other are called one hop neighbours. Multihop ad-hoc networks [1] [3] are ones in which the source node route the packets to destination node more than one hop away via intermediate nodes. Disaster management operations and battalion of soldiers are the example of applications of such cooperative ad-hoc wireless networks. This paper propose a protocol CoopGeo [1] [4], based on position location of nodes obtained using GPS technology [7].

In traditional geographic routing protocols, each node exchanges periodic one-hop "Beacons" to determine the position of its neighbours [2] [4]. Since these beacons can create severe problems in real deployments due to the highly dynamic and error-prone nature of wireless links, it uses Beacon-less geographic routing protocol (BLGR) [3]. Based on location knowledge and contention based selection processes [15] [16], the proposed protocol, aims at providing an efficient, distributed approach to select next hops and optimal operates properly with varying densities of nodes.

A geographic relay selection scheme [4] based on the knowledge of location information of nodes. By jointly combining the source-relay and relay-destination distances, the optimal relay offering the best cooperative link can be efficiently determined. However, the selection process proposed by requires a central controller to decide which relay [6] is most helpful, leading to more overhead and power consumption. 
To perform position-based unicast forwarding without the help of beacons, using contentionbased forwarding scheme (CBF) [11] the next hop is selected through a distributed contention process based on the actual positions of all current neighbours. For the contention process, CBF makes use of biased timers. The BLGF mechanism [6] is carried out using the timer's settings [11], applying an area-based assignment function. In addition, a solution to detect malicious nodes [13] normally operates during determination of a route.

Existing BLGR protocol called BOSS protocol, uses a three-way handshake [7] (DATA/RESPONSE/SELECTION) and an area-based timer-assignment function to reduce collisions among responses during the forwarder selection phase. Geographic Routing (GR) algorithms require nodes to periodically transmit HELLO messages to allow neighbours know their positions (beaconing mechanism) [9]. To reduce the control overhead due to these messages, beacon-less routing algorithms have recently been proposed. However, existing beacon-less algorithms have not considered realistic physical layers.

Other Existing protocol named CSMA/p* [16] [17], is non persistent carrier sense multiple access (CSMA) [3] with a carefully chosen non uniform probability distribution $\mathrm{p}^{*}$ that node $\mathrm{s}$ use to randomly select contention slot. It compares the optimal non-persistent CSMA, discussed possible implementation directions for the optimal protocol, and finally discussed sift. Sifts backoff distribution approximately $\mathrm{p}^{*}$ no matter how many stations are backlogged, obviating the need to track this figure with a contention window and bounded back-off.

The traditional contention access technique [11] [14] [15] is reviewed to reduce collision in networks. In traditional networks like Ethernet and WIFI when collision occurs, nodes apply the binary exponential back-off technique (BEB) to reduce collisions. In BEB fairness is the most important criteria. BEB ensures the same channel access probability for every node in the network. However this technique does not take the criteria of energy consumption. In BEB contention window size is varied according to the traffic level [15].

\section{PROPOSED COOPERATIVE CROSS-LAYER PROTOCOL}

To avoid the drawback of existing protocol, it presents a fully beaconless protocol without requiring beacons in both the greedy forwarding and recovery modes. The paper introduces the role of interactions between the MAC and physical layers and between the network and MAC layers in a cooperative scenario. The proposed cross-layer framework, called CoopGeo, consists of two joint cross-layer designs:

a. A joint network-MAC design for next hop selection and

b. A joint MAC-physical design for relay selection.

\subsection{Existing COOPMAC design with Relay solution}

Two issues, routing and relay selection, are two chief considerations.

(1) When to cooperate?

(2) Whom to cooperate with and how to do selection?

For the first question, intuitively cooperation may not be a requisite for reliable transmission if the direct link is of high quality. Therefore a cooperative MAC protocol should be carefully designed to prevent unnecessary cooperation [2]. A cooperation metric related to the instantaneous source-relay and relay-destination channel measurements was proposed in [5] to decide if cooperation is needed. 


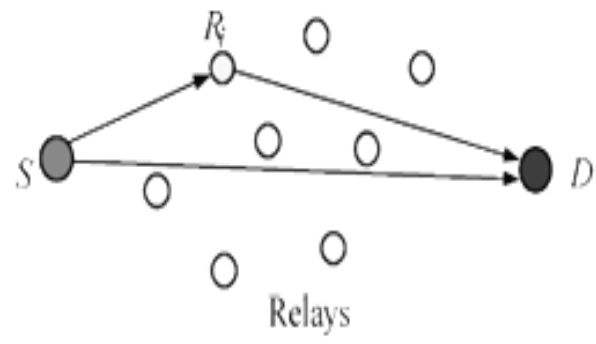

Figure1. Ad hoc network with relay selection

The second question is about cooperative MAC design addresses the typical relay selection problem. There may exist a group of available relays around the source; however, some are beneficial and some not. How to find the optimal one(s) efficiently and effectively is of vital importance to a practical MAC protocol. In particular, both the routing and relay selection solutions in CoopGeo are beaconless geographic protocols using contention-based selection processes, providing a strongly practical multi-layer integration for cooperative networks.

\subsection{Hidden and Exposed node problems}

The transmission range of stations in wireless network is limited by the transmission Power. This means that normal carrier sense mechanism which assumes that all stations can listen to each other, fails. In particular, this gives rise to hidden node and exposed node problem. A simple and elegant solution to the hidden node problem is to use small packets called RTS (Request to send) and CTS (Clear to Send) for handshaking before transmission of data packet.

\subsection{Contention-based Relay selection:}

When there is a collision in the network, it means that there are many contending transmissions in the network. In the next contending period, if every node wakes up to contend to the channel [8] [15], there would be two problems. First, the collision probability stays the same. Second, only one node can win the channel, the other nodes waste energy to wake-up and to sense the channel.

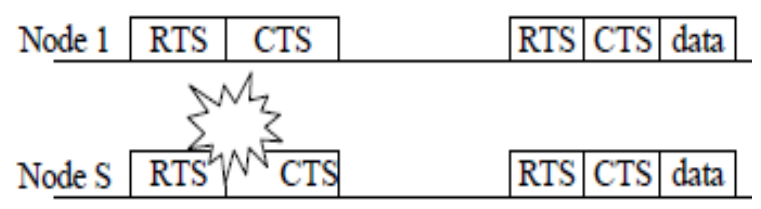

\begin{tabular}{l|l|l|l}
\cline { 2 - 3 } Node 2 & RTS & CTS & Continue to sleep \\
\hline
\end{tabular}

Figure 2. Collision avoidance with two nodes

To avoid these problems, when there is a collision, all contending sensors reduce its contending probability to a half [8]. Contending probability is the probability that sensors wake-up and go to contention period. Therefore, after a collision, a half of the contending sensors will not contend the channel in the next period as in Fig.3. They can save wake-up energy. 
Consider $\mathrm{p}$ as the contending probability of a node. This probability is always initialized to $100 \%$, which means that when a node wants to transmit a packet, it always switches its transceiver on to sense the channel. With the contending probability $\mathrm{p}$, when a node wants to send a package, it has the probability $\mathrm{p}$ to wake-up and contend the channel and a probability 1-p to stay sleeping to wait for the next period.

\subsection{Geographic Routing Protocol}

The routing process works in two phases, i.e. BLGF and BLRF. In the BLGF phase, a next hop that provides maximum progress toward the destination is selected through a timerbased contention process. As failing to find a next hop in the BLGF phase, the routing process enters transparently to the BLRF phase and applies face routing by using graph planarization along with a select-and-protest principle.

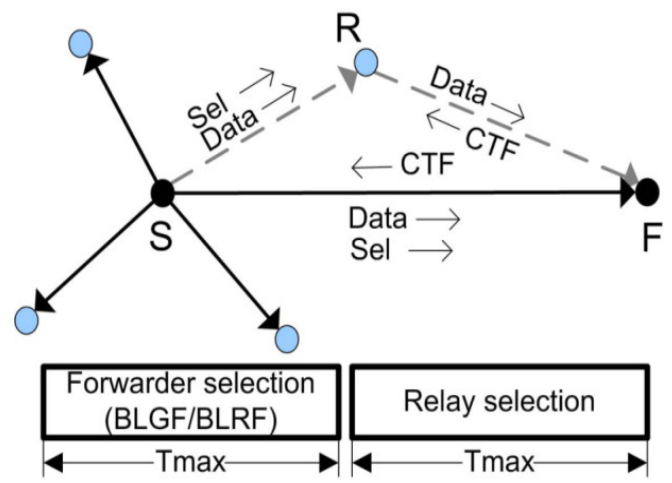

Figure 3. CoopGeo phases

Cooperative relaying is required after the routing task whenever the selected next hop decodes the data packet erroneously.

\section{PERFORMANCE METRICS}

The Selection metric [1] [3] [4] [5] is the distance parameters that depends on source-relay and relay-destination distances that indicates the SER performance [1] at the destination. The relay selection process takes place after the forwarder selection whenever the demand for cooperation is announced by a forwarder. In this case, a new contention period will be allocated for relay selection. The relay selection process, in this paper, is based on the following selection criterion [4],

$$
m_{i}=A^{2} d_{S, R i}^{p}+B d_{R i, D}^{p}
$$

where, $\mathrm{i}=1,2, . ., \mathrm{N}$ (nodes),

$\mathrm{m}_{\mathrm{i}}$ - selection metric,

$\mathrm{d}_{\mathrm{i}, \mathrm{j}}$ - distance-dependent parameters

$\mathrm{d}_{\mathrm{S}, \mathrm{Ri}}^{\mathrm{i}}-$ distance between source and Relay

$\mathrm{d}_{\mathrm{Ri}, \mathrm{D}}^{\mathrm{p}}$ - distance between Relay and destination

Here $m_{i}$ is treated as our relay selection metric, which indicates the SER performance at the forwarder-the smaller the metrics is, the better the resulting SER performance [4] will be. Therefore, the best relay can be determined according to the following criteria 
*Smaller the metrics, better the resulting SER performance.

We start with the computation of the collision probability [8] of a given contention window. Let us recall that a collision occurs if two or more nodes choose the same first slot. Consider a contention window $\mathrm{CW}$, the probability that each node chooses a slot is $1 / \mathrm{CW}$ [8]. A successful contention period is that one node chooses the first slot, and all other nodes choose later slots. The probability of collisions is in conjunction with the probability of success [9]. Hence, if there are $\mathbf{N}$ transmissions at the same time, the probability of collision $\left(\mathbf{P}_{\text {colln }}\right)$ [8] is thus calculated as

$$
\mathbf{P}_{\text {colln }}=1-\left\{(\mathrm{N} / \mathrm{CW}) \sum_{\mathrm{k}=1}{ }^{\mathrm{CW}}[(\mathrm{CW}-\mathrm{k}) / \mathrm{CW}]^{\mathrm{N}-1}\right\}
$$

Where N- No. of contending transmissions, CW- Contention window size, K- no. of slot.

In this probability of collisions when we fix the contention window $\mathrm{CW}=4$ and vary the number of contending transmissions N. Observing the expression (2), we find that the probability of collision is proportional to the number of contending transmissions. When we fix the size of the contention window $\mathrm{CW}$, if the number of contending transmissions $\mathrm{N}$ is increased, the probability of collision is increased too.

\section{SIMULATION AND RESULT ANALYSIS}

The Cooperative relay networks $n=5$ is the available relays, deployed in $R$. Denote $(x, y)$ as the coordinates of nodes. The path loss exponent is taken to be $p=2$ in these simulations and it also use QAM modulation technique. The advantage over QAM modulation technique is that it can be used to obtain both the phase shift and Amplitude modulation. This results in efficient simulation using Rayleigh channel model with constellation size varies from 4 to 512.

Table1. Simulation Settings

\begin{tabular}{|l|l|}
\hline Input & \multicolumn{1}{|c|}{ Value } \\
\hline No. of Neighbours & $1-15$ \\
Channel model & Rayleigh \\
Path Loss Exp. & 2 \\
Modulation Type & QAM \\
Constellation Size & $4-512$ \\
\hline
\end{tabular}

The neighbour nodes in the domain or outside the domain may be of maximum and so here we take 15 nodes and that can be extended to 100 nodes. The simulation result has been obtained and performance of the result is also analysed.

\subsection{Result Analysis}

The resulting figure depicts the SER versus SNR performance, where SNR is defined as P/N0 and $\mathrm{P}$ is the total transmit power fixed. Figure4. shows that the selected best relay contributes to the minimum SER at the destination as compared to other relays. 


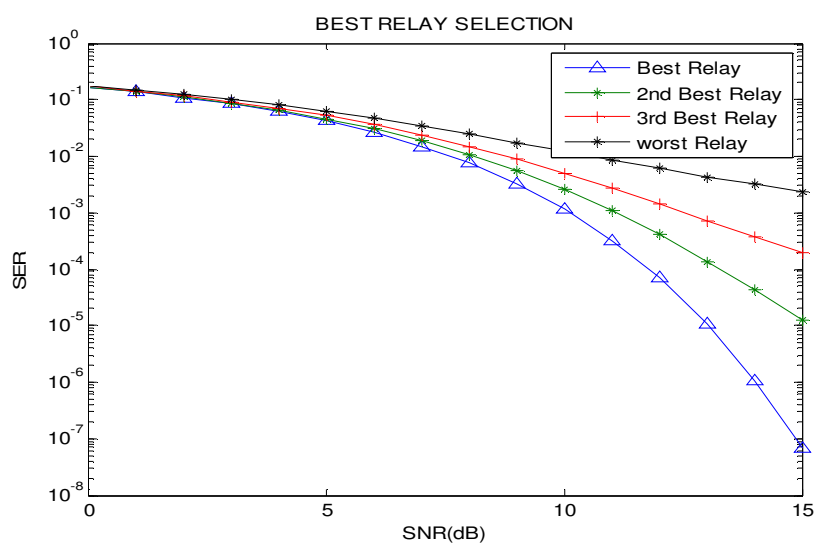

Figure4. Optimal Relay Selection

In addition, it also reveals that worst relays corresponds to larger selection metrics, that is, the smaller the selection metrics, the better the resulting SER performances. This is because each relay has the same opportunities to be selected such that the performance will be averaged over all the distributed relays.

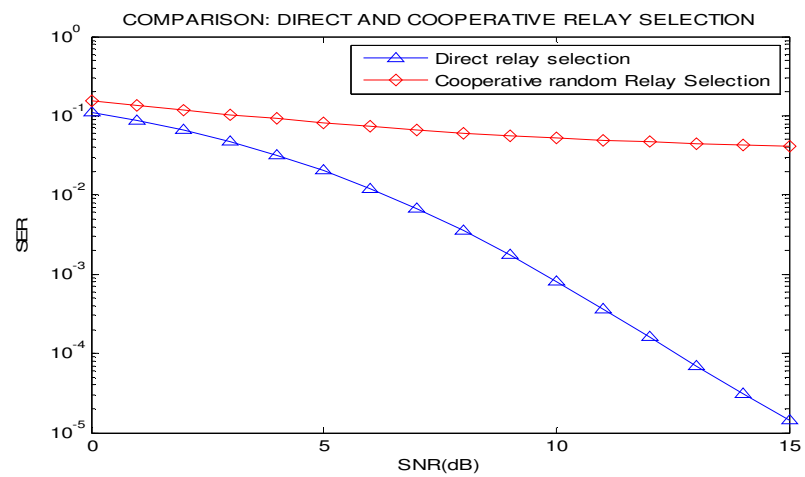

Figure5. Cooperative mode Vs Direct mode

In the Figure 5., the performance of direct transmission from the source to the destination is provided as a benchmark for a non-cooperation scheme is compared with the Cooperative relay selection scheme, which results in High Performance with the Cooperative scheme.

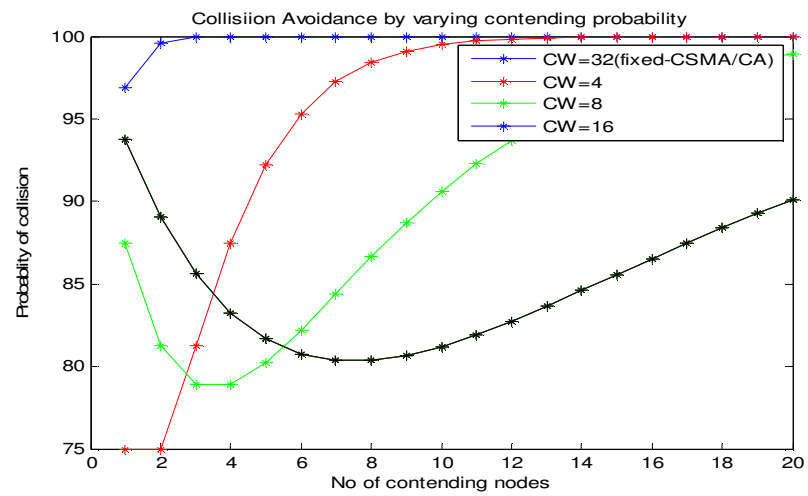

Figure6. Collision reduced -varying CW size 
The Fig.5(c), illustrates the probability of collision by using the proposal. The horizontal axis is the number of contending periods. The vertical axis is the probability of collisions for each contending period. It was observed that our approach reduces the number of collisions after a few contending periods.

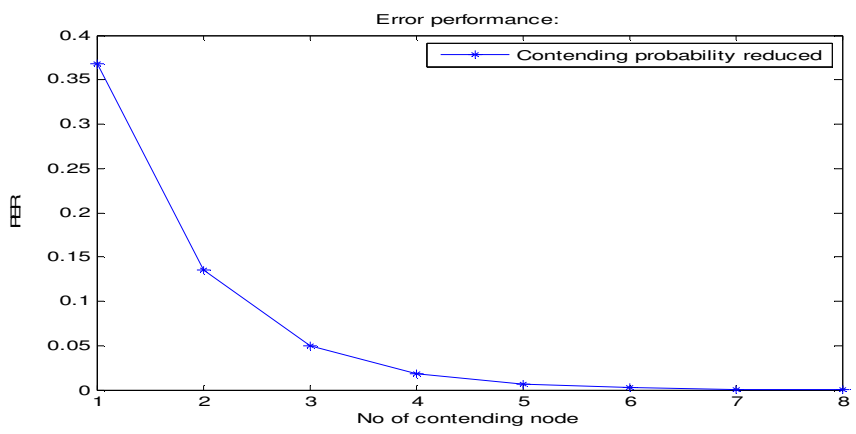

Figure7. Error performance

At the first period, the probability of collision is the same as CSMA/CA. But, after each collision, the nodes reduce their contending probability in Figure6, so that the number of contending transmission is reduce which implies a reduction of collision number.

\section{CONCLUSION}

The advantage of position-based geographic routing over other ad hoc routing protocols is the fact that nodes require only knowledge about the local neighborhood and the destination's location instead of global route topology. Therefore, position-based routing is better suited for networks with a certain degree of mobility. Thus this paper have proposed a cross-layer protocol CoopGeo based on geographic information to effectively integrate the Network, MAC, and PHY layers for cooperative wireless ad hoc networks. Simulation demonstrates that the selected best relay contributes to the minimum SER at the destination as compared to other relays. It reveals that the smaller the selection metrics, the better the resulting SER performances. Also presented and analyzed a new contention access method to reduce collisions in scalable sensor network. Proposed method uses a fixed contention window, and changes the contending probability. Error performance is also evaluated based on contention and probability error rate.

\section{REFERENCES}

[1] Teck Aguilar, Syue-Ju Syue, Vincent Gauthier, Hossam Afifi, and Chin-Liang Wang; "CoopGeo: A Beaconless Geographic Cross-Layer Protocol for Cooperative Wireless Ad Hoc Networks", IEEE Transactions on Wireless Communications, Accepted For Publication.

[2] H. Shan, W. Zhuang, and Z. Wang, "Distributed cooperative MAC for multihop wireless networks," IEEE Commun. Mag., vol. 47, no. 2, pp.126-133, Feb. 2009.

[3] J. A. Sanchez, P. M. Ruiz, R. Marin-Perez, "Beacon-less geographic routing made practical: challenges, design guidelines, and protocols,"IEEE Commun. Mag., vol.47, no. 8, pp.85-91, Aug. 2009.

[4] C.-L. Wang, S.J. Syue, "A geographic based approach to relay selection for wireless ad hoc relay networks," in Proc. 2009 IEEE Vehic.Tech. Conf. (VTC 2009-Spring), Apr. 2009, pp. 1-5.

[5] A. S. Ibrahim, A. K. Sadek, W. Su, K. J. R. Liu, "Cooperative communications with relay selection: when to cooperate and whom to cooperate with?" IEEE Trans. Wireless Commun., vol. 7, no. 7, pp. 2814-2827, July 2008. 
[6] H. Kalosha, A. Nayak, S. Ruhrup, and I. Stojmenovic, "Select-and protest-based beaconless georouting with guaranteed delivery in wireless sensor networks," in Proc. INFOCOM 2008, Apr. 2008, pp. 346-350.

[7] J. A. Sanchez, R. Marin-Perez, P. M. Ruiz, "BOSS: beacon-less on demand strategy for geographic routing in wireless sensor networks," in Proc. 4th IEEE Int. Conf. on Mobile Ad-hoc and Sensor Systems, Oct.2007, pp. 1-10.

[8] H-C. Le, H. Guyennet, N. Zerhouni, "A New Contention Access Method for Collision Avoidance in Wireless Sensor Networks", in Proc. $6^{\text {th }}$ IEEE Inter. Conf. on Networking, 0-7695-2805-8/07 -2007.

[9] P. Liu, Z. Tao, Z. Lin, E. Erkip, S. Panwar, "Cooperative wireless communications: a cross-layer approach,” IEEE Trans. Wireless Commun., vol. 13, no. 4, pp. 84-92, Aug. 2006.

[10] Kurtis Kredo IIa Prasant Mohapatra b, "Medium Access Control in Wireless Sensor Networks" 29 June 2006.

[11] P. Liu, Z. Tao, and S. Panwar, "A cooperative MAC protocol for wireless local area networks," in Proc. 2005 IEEE Int. Conf. Commun., May 2005, pp. 2962-2968.

[12] Polastre, J., Hill, J., Culler, D.: "Versatile Low Power Media Access for Wireless Sensor Networks." In: Proc. of the ACM sensys conf., Baltimore, MD (2004) 95-107.

[13] Y. C. Tay, K. Jamieson, H. Balakrishnan, "Collision-Minimizing CSMA and Its Applications to Wireless Sensor Networks", IEEE Journal on Selected Areas in Communications. Volume: 22, issue: 6, pages: 1048-1057, August 2004.

[14] M. Heissenb uttel, T. Braun, T. Bernoulli, and M. Walchli, "BLR: beacon-less routing algorithm for mobile ad-hoc networks," Computer Commun., vol. 27, no. 11, pp. 1076-1086, 2003.

[15] H. Fubler, J. Widmer, M. Kasemann, M. Mauve, and H. Hartenstein, "Contention-based forwarding for mobile ad hoc networks," Ad Hoc Networks, vol. 1, no. 4, pp. 351-369, Nov. 2003.

[16] M. Zorzi, R. R. Rao, "Geographic random forwarding (GeRaF) for adhoc \& sensor etworks: multihop performance," IEEE Trans. Mobile Comput., vol. 2, no. 4, pp. 337-348, Dec. 2003.

[17] V. Rajendran, K. Obraczka, J.J.Garcia-Luna-Aceves, "Energy -Efficient, Collision-Free Medium Access Control for Wireless Sensor Networks", Proc. ACM sensys 03, pages: 181 - 192, Los Angeles, California, 5-7 November 2003.

[18] C. E. Perkins and E. M. Royer, "Ad-hoc On Demand Distance Vector Routing," in Proc. of the 2nd IEEE Workshop on Mobile Computing Systems and Applications (WMCSA'99), 1999.

[19] Tony Larson and Nicklas Hedman, "Routing Protocols in Wireless Ad-Hoc Networks- A Simulation Study”, Master's thesis in Computer Science and Engineering 1998:362. ISSN: 1402-1617. ISRN: LTU-EX-98/362-SE.

[20] Abdelmalik Bachir and Dominique Barthel, France Telecom R\&D; Martin Heusse and Andrzej Duda, LSR-IMAG Laboratory, "Hidden Nodes Avoidance in Wireless Sensor Networks".

[21] A Tanenbaum, "Computer Networks", $4^{\text {th }}$ edition, Prentice Hall, Pearson Education 2003.

\section{Author}

G. Srimathy received B.E in Electronics and Communication Engineering from SKP Engineering College affiliated to Anna University, Chennai. Currently pursuing her M.E (Communication Systems) at S.A. Engineering College affiliated to Anna University of Technology, Chennai. She has contributed Four Technical papers in National and International Conferences and published Two Journals. Her area of interest includes Wireless Ad hoc and Sensor Networks Cooperative Communications, Cross layer design of Wireless networks, Contention-Based Collision avoidance and Network Security.

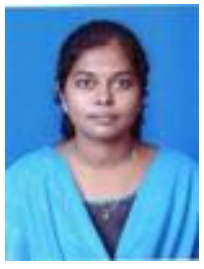

